\title{
Further investigations of random models of Uranus and Neptune
}

\author{
M. Podolak ${ }^{\mathrm{a}, *}$, J.I. Podolak ${ }^{\mathrm{b}}$, M.S. Marley ${ }^{\mathrm{c}}$ \\ ${ }^{a}$ Department of Geophysics and Planetary Sciences, Tel Aviv University, Tel Aviv, Israel \\ ${ }^{\mathrm{b}}$ Department of Computer Science, Hebrew University, Jerusalem, Israel \\ ${ }^{\mathrm{c}}$ Department of Astronomy, New Mexico State University, Las Cruces, New Mexico, USA
}

Received 19 March 1999; accepted 6 September 1999

\begin{abstract}
We present a series of computer models for Uranus and Neptune where the interior density distribution is randomly chosen. The only constraints placed on the distribution are that the density does not decrease with decreasing radius, and that the density distribution fits the observed mass and gravitational moments of these planets. Previous models of these planets all had a density discontinuity at about $70 \%$ of the total radius. We use our models to explore the space of density distributions that fit the observed gravitational moments, and set limits on the position and size of this discontinuity. We find that models are possible with no discontinuity in the mantle. In addition a density discontinuity as large as $3 \mathrm{~g} \mathrm{~cm}^{-3}$ is possible for Uranus if the discontinuity is inward of about 0.75 Uranus radii. Closer to the surface the discontinuity must be smaller. For Neptune, the larger uncertainties in the measured moments result in coarser limits on the size of the density jump. Other means of limiting the range of acceptable models are discussed. (C) 2000 Elsevier Science Ltd. All rights reserved.
\end{abstract}

\section{Introduction}

The Voyager flybys of Uranus and Neptune have measured the gravitational fields of these planets with unprecedented accuracy. Knowledge of the multipole moments of these fields, in principle, should allow for much more detailed models of Uranus' and Neptune's interiors. In practice, however, there are a number of difficulties. Perhaps the most important is that the number of possible density distributions that are consistent with the observed gravitational field is not small. As a result, it is necessary to make some a priori assumptions regarding the composition of these planets in order to reduce the number of models to a manageable level. Generally, these assumptions are based on some picture of planet formation.

For example, we would expect the materials comprising Uranus and Neptune to fall into three broad categories: (1) materials that are solid under almost all

\footnotetext{
* Corresponding author. Fax: +972-3-6409282.

E-mail address: morris@post.tau.ac.il (M. Podolak).
}

conditions of interest in the primitive solar nebula ("rock"); (2) materials that are gaseous under almost all conditions in the nebula ("gas"); and (3) materials whose phase depends on the pressure and temperature to such an extent that they are sometimes solid and sometimes gaseous ("ice"). One could then use arguments about how these materials were incorporated into the planets to set some limits on their distribution and relative abundance.

Simple models where these three categories are segregated into separate shells (with ices being allowed to mix with the $\mathrm{H}_{2}$ and $\mathrm{He}$ in the gas shell) give the result that the ices are far more abundant relative to rock than can be accounted for by any reasonable cosmogonic scenario (see, e.g. Podolak and Reynolds, 1987). As a result, a number of investigators have considered models where the ratio of ice to rock is fixed at some value consistent with cosmic abundances, while the abundance of hydrogen and helium is allowed to vary with radius in such a way as to mimic the density of an ice shell surrounded by a hydrogen rich atmosphere. A number of different models are reviewed in Podolak et al., 1991. In any case, it is clear that there 
are many plausible compositions for these planets, and there is no clear way to choose amongst them. As a result, any theoretical investigation that is based on a particular choice of composition will be limited in its generality.

A second difficulty has to do with the internal heat flow of these planets. Although Neptune, like Jupiter and Saturn, emits considerably more heat than it receives from the sun, Uranus has essentially no intrinsic heat source. This seems to imply that the internal temperatures differ from the adiabat usually assumed in such calculations (see Podolak et al., 1991 and the references therein). This too will have consequences for the computed equation of state, and as a result, for the density computed for a given pressure.

Because of these ambiguities about the behavior of material in Uranus' (and Neptune's) interior, Marley et al., 1995 (hereafter called Paper 1) decided to forego preconceived notions of what the composition should be, and look for which criteria a density distribution needed to satisfy, in order to produce a model of Uranus or Neptune which fits the observed mass, radius, and gravitational moments. Their approach was to compute random density distributions and, by analyzing those that gave fits to the observed parameters, to decide what properties characterized them.

One of the drawbacks of this work, was that a random density distribution has, by its very nature, only a small chance of fitting the observations. As a result, certain additional restrictions were placed on the models, lessening their generality somewhat. Nonetheless, the authors were able to show that the restricted set of density distributions that did fit, implied a gradual decrease in the hydrogen-helium abundance with increasing depth in the planet. As a result of the procedure used in Paper 1, the density distributions produced by this procedure all had an overall appearance similar to those found by more conventional calculations.

In this paper we continue to study random density distributions, but with the goal of finding density distributions that are very different from those studied earlier. To this end we have employed a more efficient algorithm for fitting the observational constraints. This allows a more complete mapping of the model space. Starting from a random distribution that gives the correct mass and radius, we rearrange the mass (again with a large measure of randomness), so that a given moment of inertia is reached. This guarantees that the gravitational moments will be close to those required. We thus have a much better chance of fitting those moments. The observed values for the relevant parameters are presented in Table 1. The data are taken from Hubbard et al. 1995. In Section 2 we describe the algorithm in more detail. In Section 3 we present the
Table 1

Observed parameters

\begin{tabular}{lll}
\hline Parameter & Uranus & Neptune \\
\hline Mass $\left(10^{29} \mathrm{~g}\right)$ & 0.868 & 1.024 \\
Equatorial radius $(\mathrm{km})$ & $25,559 \pm 4$ & $24,764 \pm 20$ \\
Rotation period $(\mathrm{hrs})$ & $17.24 \pm 0.01$ & $16.11 \pm 0.05$ \\
$\mathrm{~J}_{2} \times 10^{6}$ & $3516 \pm 3$ & $3538 \pm 9$ \\
$\mathrm{~J}_{4} \times 10^{6}$ & $-31.9 \pm 5$ & $-38 \pm 10$ \\
Mean density $(\mathrm{g} / \mathrm{cc})$ & 1.27 & 1.64 \\
\hline
\end{tabular}

results, in Section 4 we discuss the results, and in Section 5 we present our conclusions.

\section{The random model algorithm}

We divided up the mass distribution of the planet into a series of concentric shells. For convenience, the shells were all chosen to have equal volumes (in practice, to avoid particularly large radii for the innermost shells, some shells were given volumes of 0.25 or 0.5 of the standard volume, while others had volumes of 1.5 times that standard. These weighting factors were allowed for in the mass redistribution described below. An initial density distribution was chosen as follows. The density of the outermost shell was taken to be equal to some small value. In keeping with the spirit of this work in avoiding detailed assumptions about the composition, we chose a "generic" value of the density of the outermost shell; $10^{-4}$ of the planet's mean density. In practice, this choice did not affect the final models, since the density very quickly increased to higher values. For the Uranus models the density of the outer layer was taken to be $1.27 \times 10^{-4} \mathrm{~g} \mathrm{~cm}^{-3}$, while for Neptune a value of $1.64 \times 10^{-4}$ was chosen.

We subtracted the mass of this shell from the total mass of the planet, and the volume of the shell from the volume of the planet. We then divided the remaining mass by the remaining volume. If we assume that the density distribution inside the planet increases monotonically towards the center, the resultant density is an upper limit to the density of the next shell, $\rho_{\max }^{\mathrm{i}+1}$. The lower limit for the density is that of the previous shell, $\rho_{\min }^{\mathrm{i}+1}=\rho^{\mathrm{i}}$.

The density of the next shell was chosen to be some random value between these two extremes:

$\rho^{\mathrm{i}+1}=\rho^{\mathrm{i}}+\alpha\left(\rho_{\max }^{\mathrm{i}+1}-\rho_{\min }^{\mathrm{i}+1}\right)$

where $\alpha$ is some random number between 0 and 1 . This process was continued until all the shells but the innermost had been assigned values of density. The density of the innermost shell was chosen to have whatever value was necessary so that it contained all 
of the remaining mass. In this way an initial density distribution was chosen that guaranteed the proper mass.

In practice this process presents a small difficulty. After only a very few shells, the randomly chosen density takes on something very close to its maximal value, and from then on the density remains essentially constant. In other words, the initial models all look very similar, with a sharp density rise in the outermost layers to a constant density interior. This can be corrected by limiting the random number, $\alpha$, to something considerably less than 1 . Initially we simply cubed the number produced by our random number generator. We found, however, that the density in the outer layers still increased sufficiently quickly so that a whole class of models was omitted. In practice the random number was raised to a higher power (which was kept constant from the surface to the center for any given trial). We found that powers between 9 and 14 were sufficient to span the space of allowable models. We tried higher powers as well, but at that point, the density increased so slowly as a function of radius, that no additional models could be generated.

Although this process guarantees the correct mass, it does not necessarily give the correct gravitational moments. In order to make the search for models with the correct moments more efficient, we first tried to find models with a suitable moment of inertia. This procedure has the advantage that the computation of the gravitational moments for a given density distribution involves the numerical solution of an integral equation. This is an iterative process which is quite time consuming, and does not allow for efficient searches. The moment of inertia is much easier to compute, and so the search was carried out to find a model with this parameter correct. Afterwards the gravitational moments for the model were computed and compared with observations. Since the moment of inertia and the gravitational moments are not identical, fitting one does not guarantee fitting the other, but it does bring us into the required neighborhood. An initial mass distribution was therefore generated as described above, and the moment of inertia was computed. If it was equal to the desired value, the gravitational moments were computed. If it was not equal to the desired value, the distribution was perturbed slightly in such a way that the mass would remain constant, but the moment of inertia would move closer to the required value.

The mass rearrangement was achieved by randomly choosing both the inner and outer radii of the region from which mass was to be moved. The initial moment of inertia was always found to be higher than the desired value, so the mass of each of the shells in this region was reduced by a small, prescribed, amount. The same amount of mass was added to an equal number of shells starting from the center. Because the shells had equal volumes (after allowing for the weighting factors described above), the total mass remained unchanged. This process was repeated iteratively until the desired moment of inertia was achieved. At this stage we had a model with a density distribution that both gave the correct mass, and a reasonable moment of inertia. The gravitational moments were computed using a level surface approach (Zharkov and Trubitsyn, 1978). The expansions were carried out to third order (up to and including $\mathrm{J}_{6}$ ). More details are given in Paper 1. If the gravitational moments were not within the error bars of the observed values, a new initial distribution was generated.

There is an additional advantage to this method. For a given initial mass distribution, we can vary the moment of inertia to which the model is a fit. In this way it is possible to perturb an initially random density distribution and explore a range of models derived therefrom, in particular those with a desired $\mathbf{J}_{2}$. Although it may be argued that the procedure of fitting to the moment of inertia necessarily removes, to some degree, the randomness of the mass distribution, the fact remains that we were able to produce models which fit all of the observed parameters, and which had density distributions which differed both from one another and from any previously published distributions. Since our main objective was to explore the range of possible models, the efficiency of this algorithm more than compensates for its being less than truly "random". We present some of these models below.

\section{Model results}

We produced some 2000 models of Uranus with a moment of inertia in the expected range. Of these, some 150 matched the observed values of the gravitational moments to within the published error bars. This is a significant improvement over the success rate in Paper 1, although it was achieved at the expense of first "massaging" the initial density distribution to get a reasonable moment of inertia. In addition, since in many cases, the same initial distribution was run several times for different moments of inertia, a direct comparison of our statistics to those of Paper 1 is not possible.

While the $\mathrm{J}_{2}$ of a great many models was correct, their $\mathrm{J}_{4}$ was too negative. This is due to the fact that $\mathrm{J}_{4}$ is particularly sensitive to the density of the outermost layers, while $\mathbf{J}_{2}$ is sensitive to a larger volume of the planet (see e.g. Hubbard, 1974). Smooth redistribution of the mass allowed our code to converge to a desired $\mathbf{J}_{2}$, but the density distribution almost always 
had too much mass in the outermost layers, and the absolute value of the associated $\mathrm{J}_{4}$ was always too high.

In order to lower the absolute value of $\mathrm{J}_{4}$, a density discontinuity was introduced in Paper 1. This discontinuity was in addition to the one marking the boundary between the high density region at the center of the planet (core) and the surrounding region (envelope), and usually appeared at about 0.7 planetary radii. More standard models also found that such a discontinuity (or at least a very rapid density change with radius) was necessary for fitting the observed moments (see Podolak et al., 1991; Wisdom, 1997). We therefore allowed for such a discontinuity as well. The method for introducing this discontinuity was as follows. A shell was chosen at the place where we wished to introduce a discontinuity. When iterating to the desired value of the moment of inertia the mass was rearranged as before, but only a fraction, $f$, of the mass to be moved was rearranged in this way. The rest was distributed evenly among all the mass shells interior to the chosen shell. This causes a density discontinuity at the chosen shell whose size is proportional to $(1-f)$. In this way we were able to explore the effect of varying both the position of the discontinuity and its magnitude.

Fig. 1 shows a series of models for a density jump at a radius of 0.79 Uranus radii. Plotted are $\mathbf{J}_{2}$ and $\mathbf{J}_{4}$ for each of the models along with the observational limits for these parameters (vertical solid line). The models belong to five classes having density jumps at $r / R=0.79$ of 0.13 (double triangles), 0.78 (circles), 2.08 (pluses), 2.21 (rectangles), and 2.47 (triangles) $g$ $\mathrm{cm}^{-3}$ respectively. As can be seen from the figure, models with a jump of $2.21 \mathrm{~g} \mathrm{~cm}^{-3}$ and greater consistently have too high a $\mathbf{J}_{2}$. There is too much mass in the outer layers, and one cannot get models with a low

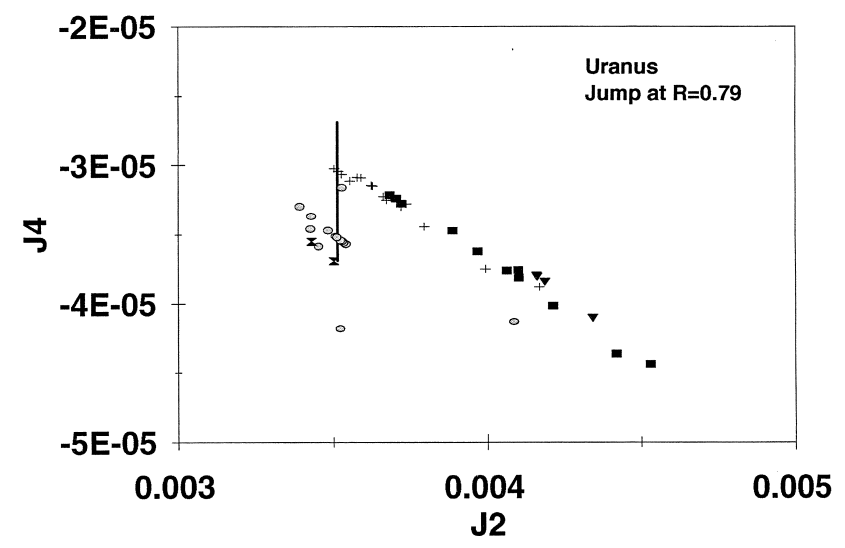

Fig. 1. $\mathbf{J}_{4}$ as a function of $\mathbf{J}_{2}$ for random models computed with a discontinuity at $r / R=0.79$ for density jumps of 0.13 (double triangles), 0.78 (circles), 2.08 (pluses), 2.21 (rectangles), and 2.47 (triangles) $\mathrm{g} \mathrm{cm}^{-3}$ respectively. enough $\mathbf{J}_{2}$. In addition, $\mathbf{J}_{4}$ is too negative. A density jump of $2.08 \mathrm{~g} \mathrm{~cm}^{-3}$ is barely sufficient, while smaller discontinuities, even as low as $0.13 \mathrm{~g} \mathrm{~cm}^{-3}$, also give models that fit the observations. As can be seen from the figure, only a small fraction of the models computed, fit the moments to within the observed error bars. Other choices for the position of the discontinuity gave similar results. In what follows we discuss only those models which fit all the observed parameters to within the error bars.

There are two things that must be kept in mind with regard to this discontinuity. The first is that although our method generates a discontinuity at the position we prescribe, it does not prevent other discontinuities from appearing as a result of the randomness of the distribution. The second point is that although a particular model may not have a formal discontinuity in the density, there may still be a rapid, but continuous, increase in the density. Models of this type have been computed for a realistic equation of state by Stevenson (see Podolak et al., 1991), and for a model with a density distribution described by Chebyshev polynomials (Wisdom, 1997). Fig. 2a shows the density distri-

(a)

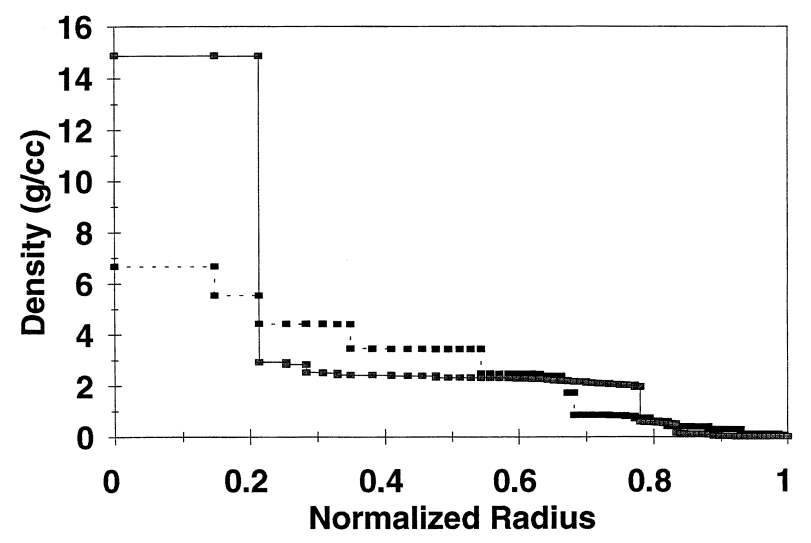

(b)

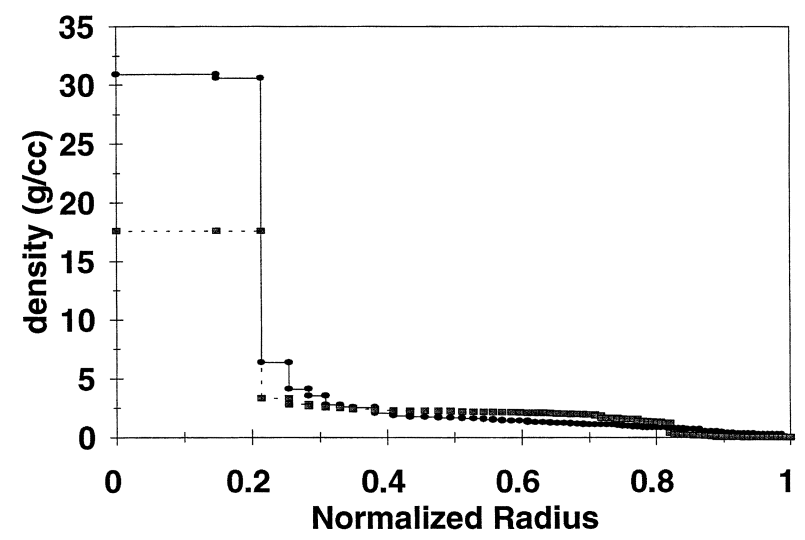

Fig. 2. (a) Density as a function of radius for two Uranus models where no density discontinuity was assigned. (b) Two additional models of Uranus with no assigned density discontinuity. 
butions for two models of Uranus for which we have prescribed a zero (i.e. no) discontinuity in density. Both models fit the observed gravitational harmonics. As can be seen from the figure, there are still considerable discontinuities due to the randomness in the choice of the original distribution. Thus prescribing a zero discontinuity is not a guarantee that the density distribution will, in fact, be continuous.

Fig. $2 b$ shows an additional two models which fit the gravitational moments. The one with the light squares still has a slight density jump at $r / R=0.82$, while the one marked by the dark ovals has an essentially continuous density distribution until a normalized radius of about 0.3 is reached. This latter model shows that the gravitational moments alone do not require a density discontinuity in the envelope, and a continuous envelope can be consistent with the observed moments.

The slow increase in density through the envelope has another consequence. The envelope does not contribute sufficient mass to the planet, and this shortage must be made up by a dense core. In this particular case, the core density is $31 \mathrm{~g} \mathrm{~cm}^{-3}$ at the center. This is quite high, and corresponds to the density of olivine at a pressure of $\sim 130 \mathrm{Mbar}(13 \mathrm{TPa})$. Pure iron would give such a density at $\sim 65 \mathrm{Mbar}(6.5 \mathrm{TPa})$, and pure gold at $\sim 4$ Mbar (400 GPa). Certainly considerations based on the equations of state of reasonable materials under high pressure can be used to further winnow out unlikely models, but at this stage we merely wish to map out the extremes of the density distributions which can fit the observed gravitational moments.

Fig. 3 shows the limits we have found for the maximum density jump for models of Uranus (x) and Neptune $(+)$. Although we cannot claim to have exhaustively mapped the space of possible models, we feel that these boundaries are representative of the actual limits on possible distributions for the interiors of these planets. We have plotted only the region

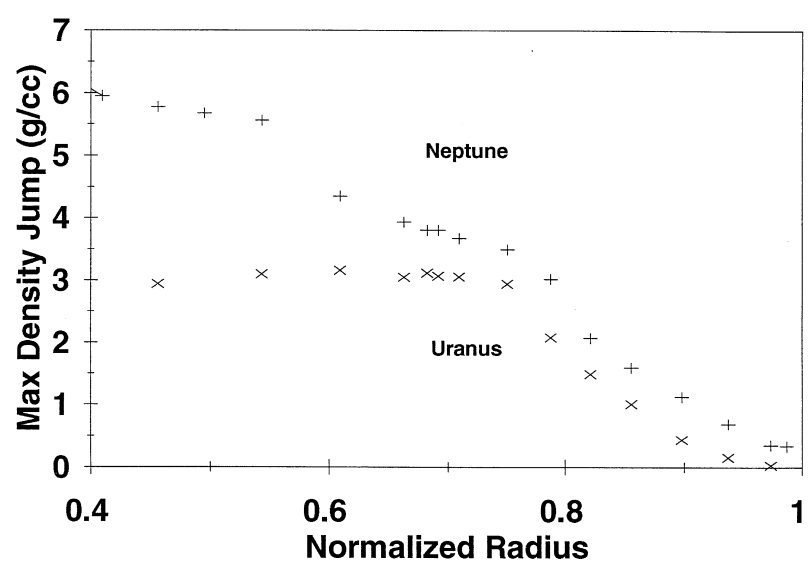

Fig. 3. Upper limit of the allowed density discontinuity as a function of position. For both Uranus and Neptune the lower limit is zero. $0.4<r / R<1$ in order not to confuse the density jump in the envelope with the possible presence of a core. The error bars on the observed values of both $\mathrm{J}_{2}$ and $\mathrm{J}_{4}$ for Neptune are considerably larger than those for Uranus, so the limits on the allowed density jump are larger as well. In addition, because of the uncertainty in $\mathrm{J}_{2}$ for Neptune, the region inward of $r / R=0.6$ is not well constrained. This explains why the maximum density jump continues to increase for decreasing $r / R$. For Uranus, however, where the value of $J_{2}$ is very well constrained, there actually appears to be a slight downturn in the maximum allowed density jump. This seems to be due to the fact that for a density jump deep in the envelope, the larger the jump, the less mass is left in the outer envelope. In order to keep the total mass of the planet equal to the observed value, the density can only decrease slowly as $r / R$ approaches 1 . As a result, the density in the outermost layers is too high, and $\mathrm{J}_{4}$ is too negative. Thus too large a discontinuity is precluded.

Once we get sufficiently close to the center, the additional mass added by the discontinuity is not large enough to affect the outer layers, and a large discontinuity (the core) is allowed. Presumably, we will see a similar effect for Neptune when its gravitational moments are more precisely determined. At large normalized radii there is also a downturn in the maximum value of the density discontinuity. This is due to the fact that near the surface the density is of necessity low, and a large jump is simply not possible.

\section{Discussion}

For the random models of Uranus and Neptune which we have computed, the interior of Uranus is much better constrained, and we have been able to set interesting limits on the size and position of any sharp

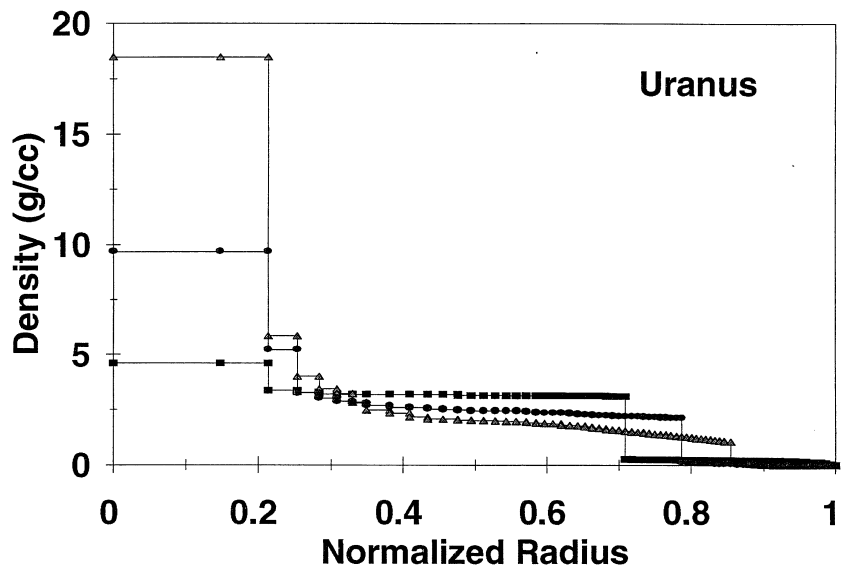

Fig. 4. Three density distributions for Uranus that fit the mass and gravitational moments. 
change in the density distribution of the interior. Similar limits can be set for Neptune, but they are much less restrictive. The question then becomes whether there are any other criteria that might allow us to eliminate some of these possibilities as unphysical, and thus further constrain the parameter space.

We have already seen that one possible approach is to exclude all those models with too high a central density. Clearly some additional work is needed to clarify exactly what limits are "reasonable" for this parameter. A second possibility can be seen in Fig. 4. Here we have three models of Uranus which fit the observed parameters. One model, marked by rectangles, has a central density that is comfortably low, perhaps even too low. But what is more of a problem is the large region of constant density. Since the pressure must increase as $r$ decreases, there will be a very large change of pressure over which the density will remain constant. This could only happen if the temperature increased very sharply throughout this region.

The pressures associated with a particular density distribution can be computed by integrating the equation of hydrostatic equilibrium:

$\frac{d P}{d r}=-\frac{\mathrm{G} M(r) \rho}{r^{2}}$

and that of mass conservation:

$\frac{d M}{d r}=4 \pi r^{2} \rho$

In the above, $P(r)$ is the pressure at a given radius, $\rho(r)$ is the density at that point, and $M(r)$ is the mass contained in a sphere of radius $r$. Fig. 5 gives the pressure-density relationships for the models shown in Fig. 4. Also shown are curves for "rock", "ice", and an adiabat through solar composition material. The

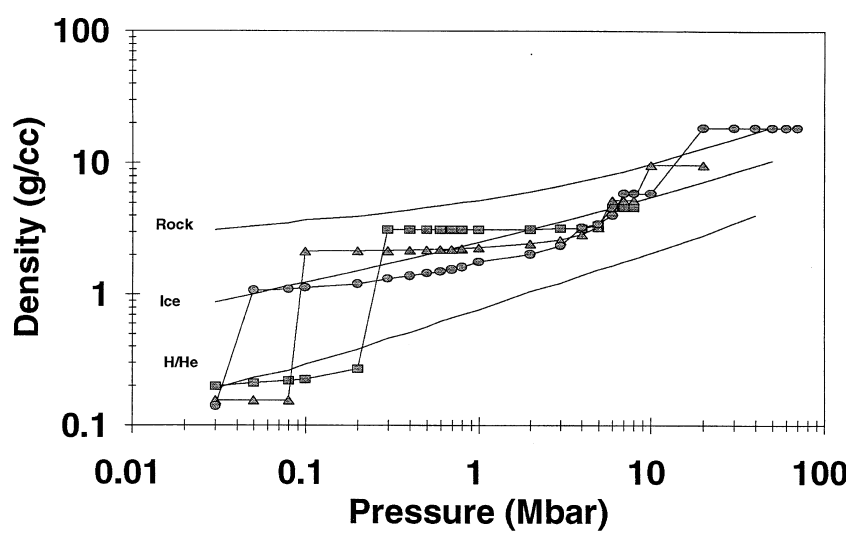

Fig. 5. Pressure-density relationships for the three models shown in Fig. 4. The symbols designating the different curves correspond to the symbols of Fig. 4. The three solid curves are the equations of state for rock, ice, and an adiabat through a solar mix (marked $\mathrm{H}$ / $\mathrm{He})$. equations of state for "rock" and "ice" are taken from Podolak and Cameron (1974) and are for zero temperature. Unless the temperatures in the interiors of Uranus and Neptune are very high indeed (see below), the thermal contribution to the equation of state should be small. The equation of state for the solar composition adiabat is for a 1-bar temperature of $70 \mathrm{~K}$, and is computed according to the prescription given in Podolak and Cameron (1974).

For the model designated by the rectangles, a computation of the pressure-density relationship shows that the pressure changes from 0.3 Mbar to 4 Mbar while the density changes from 3.1 to $3.2 \mathrm{~g} \mathrm{~cm}^{-3}$. For comparison, at zero temperature, a typical "rock" changes its density from 4.2 to $7.3 \mathrm{~g} \mathrm{~cm}^{-3}$ while a solar mix of $\mathrm{H}_{2} \mathrm{O}, \mathrm{NH}_{3}$, and $\mathrm{CH}_{4}$ changes its density from 1.8 to $4.0 \mathrm{~g} \mathrm{~cm}^{-3}$ in this pressure range.

A sharp temperature rise would account for this behavior, but it would have to be very sharp indeed. If we assume that the temperature at $0.3 \mathrm{Mbar}$ is low, then a density of $3.1 \mathrm{~g} \mathrm{~cm}^{-3}$ corresponds to a mix of $30 \%$ ice by mass and $70 \%$ rock. Assuming that this mixture remains constant, and the density rises to $3.2 \mathrm{~g}$ $\mathrm{cm}^{-3}$, the temperature would have to rise to approximately $10^{5} \mathrm{~K}$ in order to provide the appropriate pressure. Such a large temperature gradient should give a much larger heat flux than is currently observed for Uranus, and this would seem to be grounds for eliminating this particular model as a representation of Uranus' interior.

The pressure-density relationships for the other two models are also shown. The one represented by triangles also shows a large range of pressure over which there is a negligible density change, while the curve marked by the ovals behaves more like ordinary materials (except at the very highest pressures), and may

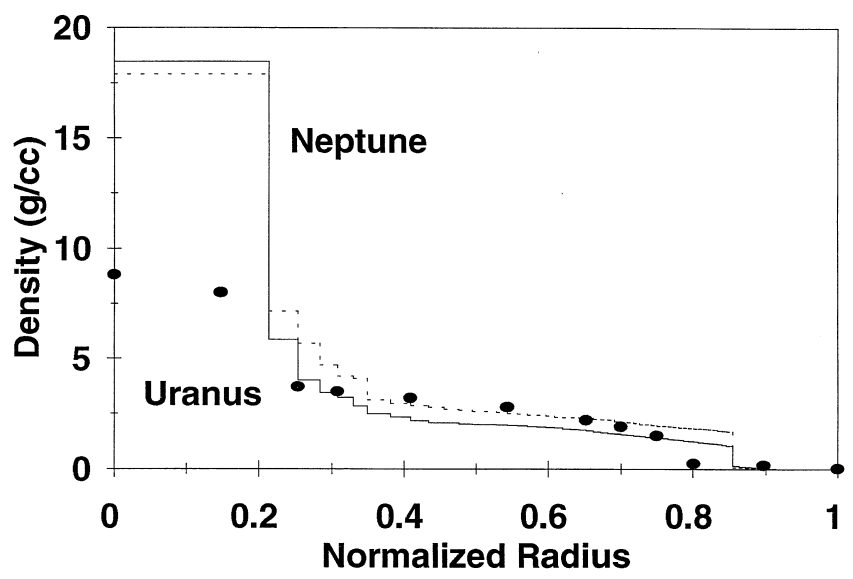

Fig. 6. Density distribution for a Uranus (solid curve) and a Neptune (dashed curve) model which fit the mass and gravitational moments, and have similar interior densities. The solid ovals are density-radius points for a Uranus model computed based on realistic equations of state. 
be within acceptable limits. Further elaboration of the limits that can be placed on the behavior of the equation of state should allow us to exclude additional candidate models. Such studies will allow us to interpret those density distributions that are physically plausible. These ideas will be pursued in future work.

One additional method of limiting the number of acceptable models is by assuming that Uranus and Neptune have similar internal structures. This is, admittedly, a debatable criterion, since the planets have very different heat flows. Still it might be reasonable in view of the similarity of their radii, masses, and location in the solar system, and we present it merely as a possibility. With such an assumption, we need only consider those density distributions that appear in models for both Uranus and Neptune. An example of such a pair is shown in Fig. 6. The solid curve is the density distribution for Neptune, while the dashed curve is for Uranus. For comparison, a density distribution for Uranus computed based on a standard equation of state is also shown (dots). Fig. 7 shows the pressure density relation for the two models shown in Fig. 6. Also plotted are pressure density curves for "rock", "ice" and a solar mix of hydrogen and helium. As can be seen, the density distributions in both planets approximate those expected for realistic materials, although the Neptune curve is probably too flat. If no model of Neptune can be found which both has reasonable equations of state, and has a density distribution similar to the Uranus model, one might argue that these are grounds for eliminating the Uranus model as well.

There is another piece of analysis that can be done on these models. We have computed the quantity $\rho d P / d \rho$ at zero temperature for the substances: $\mathrm{H}, \mathrm{He}$, $\mathrm{CH}_{4}, \mathrm{H}_{2} \mathrm{O}, \mathrm{C}, \mathrm{CO}, \mathrm{O}, \mathrm{SiO}_{2}, \mathrm{Si}, \mathrm{Fe}_{2} \mathrm{SiO}_{4}, \mathrm{FeO}$, and $\mathrm{Fe}$ for pressures between 1 and 100 Mbar, using the equations of state of Salpeter and Zapolsky (1967) and

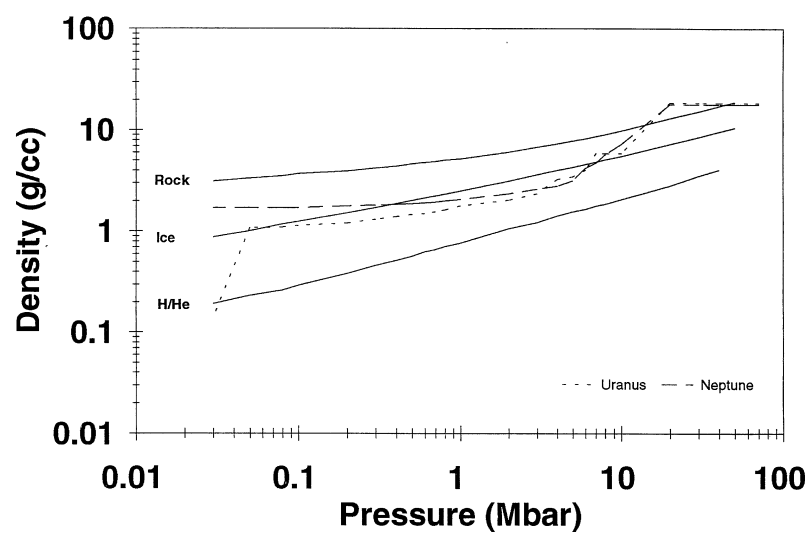

Fig. 7. Pressure-density relationships for the distributions shown in Fig. 6. Also shown are the equations of state for rock, ice, and a solar adiabat.
Zharkov et al. (1978). Although both the density and its pressure derivative differ between the two equations of state, the product of the two is remarkably constant. For the pressure range cited, this product stays constant to within a factor of a few for each of the substances listed, although the value of this product does increase with increasing molecular weight of the molecule. The values are given in Table 2. Using this fact we can argue as follows: We define the mean density at a point inside the planet via

$\langle\rho(r)\rangle \equiv \frac{3 M(r)}{4 \pi r^{3}}$

Then, solving for $M(r) / r^{3}$ and substituting this into Eq. (2) gives

$\frac{d P}{d r}=-G \frac{4 \pi}{3} r\langle\rho(r)\rangle \rho(r)$

and, assuming that the equation of state is independent of temperature

$\frac{d \rho}{d r}=\frac{d P}{d r} \frac{d \rho}{d P}=-\frac{4 \pi G}{3}\langle\rho(r)\rangle r \rho(r) \frac{d \rho}{d P}$

Now for a monotonic density distribution, $\langle\rho(r)\rangle \geq \rho(r)$. It is also true that $\langle\rho(r)\rangle \geq\langle\rho(R)\rangle$, where $R$ is the radius of the planet. This allows us to put a lower limit on $|d \rho / d r|$, namely

$\left|\frac{d \rho}{d r}\right| \geq \frac{4 \pi G}{3} r \rho \frac{d \rho}{d P} \max \{\rho(r),\langle\rho(R)\rangle\}$

If the density distribution is given (by the random models presented here, for example), and one can make some reasonable guess as to the composition, then at any point in the planet we can set a lower limit on the slope of the density distribution near that point. The important caveat here is that we must assume that the temperature is low enough so that the thermal contribution to the pressure can be neglected.

Table 2

Values of $\rho d P / d \rho$ for different substances

\begin{tabular}{lrr}
\hline Substance & Mean atomic weight & \multicolumn{1}{c}{$\rho d P / d \rho$} \\
\hline $\mathrm{H}$ & 1.01 & $1.5-2 \times 10^{-13}$ \\
$\mathrm{He}$ & 4.00 & $0.9-1 \times 10^{-12}$ \\
$\mathrm{CH}_{4}$ & 3.21 & $0.8-1.4 \times 10^{-12}$ \\
$\mathrm{H}_{2} \mathrm{O}$ & 6.01 & $1.5-3 \times 10^{-12}$ \\
$\mathrm{C}$ & 12.01 & $1.5-2.5 \times 10^{-12}$ \\
$\mathrm{CO}$ & 14.01 & $3-6 \times 10^{-12}$ \\
$\mathrm{O}$ & 16.00 & $2-3 \times 10^{-12}$ \\
$\mathrm{SiO}$ & 20.03 & $2-8 \times 10^{-12}$ \\
$\mathrm{Si}$ & 28.1 & $3-6 \times 10^{-12}$ \\
$\mathrm{Fe}$ & 29.1 & $0.8-1.8 \times 10^{-11}$ \\
$\mathrm{FeO}$ & 35.9 & $0.4-1.8 \times 10^{-11}$ \\
$\mathrm{Fe}$ & 55.8 & $0.5-1.6 \times 10^{-11}$ \\
\hline
\end{tabular}


This should be true deep in the planet's interior, where the pressures are sufficiently high.

As an example of the effect of temperature in these regions, at $1 \mathrm{Mbar}$ for $\mathrm{Fe}$ at $T=0$, the SalpeterZapolsky (SZ) equation of state gives a density of $8.10 \mathrm{~g} \mathrm{~cm}^{-3}$. At a temperature of $10^{4} \mathrm{~K}$, using a simple Debye model, we can estimate the thermal contribution to the pressure as $P_{\mathrm{T}}=200 \mathrm{kbar}$. Keeping the total pressure constant at $1 \mathrm{Mbar}$, and lowering the zero-temperature pressure to $800 \mathrm{kbar}$, lowers the density to $7.75 \mathrm{~g} \mathrm{~cm}^{-3}$, a correction of some $4 \%$. At 10 Mbar and $10^{5} \mathrm{~K}$, the correction to the density is $13 \%$. In both cases the expected temperatures at these pressure levels are much lower. For water, the corrections at these pressures and temperatures are $5 \%$ and $18 \%$ respectively. In other words, thermal effects on the density should be of the order of $10 \%$. On the other hand, the value of $\rho d P / d \rho$ at a particular pressure can differ from the mean in Table 2 by as much as a factor of 2 .

We can now apply this criterion to the Uranus model shown in Fig. 7. In the "core" region, the density at the edge of the core is $18.5 \mathrm{~g} \mathrm{~cm}^{-3}$, and if we take a value of $\rho d P / d \rho=10^{-11}$ dynes $\mathrm{cm}^{-2}$, consistent with $\mathrm{Fe}$ and other core-forming material, the density at the very center must be at least $19.0 \mathrm{~g} \mathrm{~cm}^{-3}$. This is not terribly different from the assumption of constant density, so that the model is self-consistent in this sense. On the other hand, if we look at the region between 0.53 and 0.85 planetary radii, the density should increase much faster than it does. For $\rho d P / d \rho=10^{-11}$, and a density at $r=0.85 R$ of $1.07 \mathrm{~g}$ $\mathrm{cm}^{-3}$, the density at $\mathrm{r}=0.53 \mathrm{R}$ should be at least $6.03 \mathrm{~g}$ $\mathrm{cm}^{-3}$. The model gives 1.99. This is too large a difference to be accounted for by uncertainties in the value of $\rho d P / d \rho$. On the other hand, if we take $\rho d P / d \rho=$ $2 \times 10^{-12}$ which is more consistent with "ice", the theoretical density at this point reduces to 2.06, a difference which is easily accounted for by the uncertainty in $\rho d P / d \rho$.

For the Neptune model in Fig. 7, a similar analysis gives an increase across the core of $0.6 \mathrm{~g} \mathrm{~cm}^{-3}$. This too is small compared with the density of the core. Between $r=0.51 R$ and $r=0.77 R$, the density should increase from 1.9 to at least $6.6 \mathrm{~g} \mathrm{~cm}^{-3}$ for "rock" and to at least $2.8 \mathrm{~g} \mathrm{~cm}^{-3}$ for "ice". The actual model value is $2.6 \mathrm{~g} \mathrm{~cm}^{-3}$, too low for either composition. This could be indicative of a temperature effect, or of the fact that the molecules comprising this layer have a mean atomic weight even lower than that of water. If we wish to assume that both Uranus and Neptune have similar compositions in this region, then it seems that Neptune's interior must be hotter here. Perhaps this is a clue to why Neptune has an internal heat source while Uranus does not. Detailed analyses along these lines should help to provide insights for a more judicious choice of compositional models to explore in detail.

\section{Conclusions}

We have computed models of Uranus and Neptune based only on the assumption that the density distribution in these planets is monotonic, and the requirement that it fits the observed mean density and the gravitational moments, $\mathbf{J}_{2}$ and $\mathbf{J}_{4}$. This procedure produces density distributions significantly different from those computed from an a priori choice of composition. We have shown how general properties of the equation of state may be used in conjunction with these models to draw inferences about possible compositions. In addition, by exploring the space of density distributions that fit the observational constraints, we have been able to place limits on the size and position of possible discontinuities in the density. Aside from the obvious density discontinuity at the core, we found that it is possible to construct models of both Uranus and Neptune that had no additional density discontinuity in the envelope. This includes models with a much smoother density distribution in the envelope than has previously been computed (Fig. 2b), although the equations of state represented by these density distributions most probably do not refer to realistic materials.

These smoother models have an additional significance in that they are related to the maximum amount of hydrogen and helium that can be put into these planets. A given model density profile can be achieved with a variety of compositions. In particular, we can view it as a mixture of "rock" and "ice" in solar proportions combined with hydrogen and helium "gas". The same profile can be seen as containing even more "gas" if we interpret it as a mixture of only "rock" and "gas". Because of the high compressibility of the "gas" relative to the "rock" and "ice", those models which can be seen as having more "gas" in the deeper layers will be interpreted as having a higher overall "gas" content. In other words, we expect models without a density discontinuity (i.e. models which do not have a clear demarcation between a region of high "gas" content and a region of low "gas" content) to allow an interpretation with the most hydrogen and helium. This is indeed borne out in numerical tests on the models. If we assume that the envelope is everywhere composed of a mixture of "rock" and "ice" in solar proportions, and whatever amount of "gas" (hydrogen and helium in the solar ratio) is required to reproduce the density profile, then we find that the maximum gas content (hydrogen + helium in the solar ratio) for Uranus is about 4.2 Earth masses, and about 3.2 Earth masses for Neptune. If we assume that the 
model envelope is composed of only "rock" and "gas", we can get somewhat higher values; 5.0 Earth masses for Uranus and 4.7 Earth masses for Neptune. These are the upper limits to the hydrogen-helium contents for these planets.

An additional possibility for extracting interesting models from the set we have computed is to insist that the overall compositions of both Uranus and Neptune be sufficiently similar. In this way we can search for model pairs with realistic equations of state. The pair of such models presented above suggests that interior temperatures in Neptune may be higher than in Uranus. This might help explain the differences in the observed internal heat source.

While we cannot claim that our procedure gives an exhaustive picture of the entire space of possible models, it has allowed us to find density distributions which fit the observed gravitational moments, are physically plausible, and are very different from those previously published. Hopefully, continued study along these lines will give new insights into the possible structure and composition of Uranus and Neptune.

\section{Acknowledgements}

We gratefully acknowledge the suggestions of Dr W. Hubbard and an anonymous referee, which significantly improved this manuscript.

\section{References}

Hubbard, W.B., 1974. Inversion of gravity data for giant planets. Icarus $21,157-165$.

Hubbard, W.B., Pearl, J.C., Podolak, M., Stevenson, D.J., 1995. Interior of Neptune. In: Cruickshank, D. (Ed.), Neptune and Triton. University of Arizona Press, Tucson, pp. 109-138.

Marley, M.S., Gomez, P., Podolak, M., 1995. Monte Carlo interior models for Uranus and Neptune. J. Geophys. Res. 100, 23,34923,353 .

Podolak, M., Cameron, A.G.W., 1974. Models of the giant planets. Icarus 22, 123-148.

Podolak, M., Reynolds, R.T., 1987. The rotation of Uranus, its internal structure and the process of planetary accretion. Icarus 70 , $31-36$

Podolak, M., Hubbard, W.B., Stevenson, D.J., 1991. Models of Uranus' interior and magnetic field. In: Bergstralh, J., Minor, E., Matthews, M.S. (Eds.), Uranus. University of Arizona Press, Tucson, pp. 29-64.

Salpeter, E.E., Zapolsky, H.S., 1967. Theoretical high-pressure equations of state including correlation energy. Phys. Rev. 158, 876-886.

Wisdom, J., 1997. Non-perturbative Hydrostatic Equilibrium. Preprint.

Zharkov, V.N., Trubitsyn, V.P., 1978. Physics of Planetary Interiors. Pachart Press, Tucson.

Zharkov, V.N., Tsarevsky, I.A., Trubitsyn, V.P., 1978. Equations of State of Hydrogen, Hydrogen Compounds, Crystals of Inert Gases, Oxides, Iron, and FeS. NASA TM-75311. 